\title{
An improved ant colony foraging behavior modeling in the application of robot path planning problem
}

\author{
Jian Peng ${ }^{1, \text { a }}$, Zhiwei Bao ${ }^{2, b}$ \\ ${ }^{1}$ School of Automation, China University of Geosciences, Wuhan 430074, China;
}

Keywords: foraging behavior model; complex adaptive system; agent.

\begin{abstract}
In order to study and apply the ant foraging theory,this paper introduced the modeling method based on agent to construct the ant colony foraging behavior model. Through constructing many effective ant behavior rules,such as the path selection rules, which was added the function of perceiving information, the reward and punishment rules which could regenerate the pheromone and strengthen the speed and the intelligence of ant routing. Comparing with the existing classic literature,it determined the effectiveness of the model. In order to verify the adaptability of ants foraging behavior model,the model was used to the local path planning of mobile robot. The simulation results show the ants foraging behavior model can quickly determine the optimal path in the complex environment.
\end{abstract}

\section{Introduction}

The principle of ant colony optimization theory is between ant colony to food source and nest through indirect exchanges and cooperation to find the most short-circuit foraging. The simulation to the process of ant colony foraging behavior has become an important step in the study of ant colony optimization theory. Ant colony foraging model simulation research was first designed by simulation software based on. net Wilensky Logo, used to simulate the real ants foraging behavior of nature. Ants foraging model based on Bonabeau is also presented,designed and implemented by domestic scholars, the effectiveness and adaptability of the ant colony algorithm is verified by the multi-agent simulation system. In this paper, based on the ant colony foraging for food source of system modeling and simulation, using the bottom-up modeling method of the agent dynamically to show the whole process of the ants foraging and determine the validity of the model in the experiment ${ }^{[1-3]}$.

Mobile robot path planning is the basis of the research on robot control system. The traditional path planning methods have their own defects, it becomes a research hotspot to seek a better algorithm in the field. This paper search path of robots based on ant colony foraging model and similarity, the ant colony foraging model is used to solve local robot path planning in the unknown environment, through setting ant colony foraging behavior rule in grid environment more effectively, obtained the optimal path in a complicated environment ${ }^{[4-6]}$.

\section{Ant colony foraging behavior model and simulation is established based on agent}

\subsection{Ant colony foraging behavior and complex adaptive system theory}

Adaptive complexity is the core idea of Complex Adaptive System (CAS) theory, This theory emphasizes the subject is a "live" with initiative and adaptability of the entity, Main body in the process of foraging ants have intelligence, adaptability and initiative, ants' path is marked by pheromones, but does not necessarily move to the direction of the largest pheromone,so it is random. As can be seen from the above analysis, the connotation of the concept of CAS and the characteristics of ants foraging behavior is the same. So we can take advantage of CAS theory research methods to study ants foraging behavior. CAS theory provides a bottom-up modeling methods, and through the simulation to the evolution of the complex real world phenomenon, called the agent based modeling and simulation method.The agent, as a basic component of the model, by setting the rules of the behavior of the agent for the characteristics of evolution for system, the main research method is one of the CAS theory ${ }^{[7]}$. 


\subsection{Model environment and environment parameter description}

The space of ants is a virtual world of the abstract, it needs a nest, food source, other ants and obstacles in this space, the pheromone information should be provided in the environment. The model of information known as two kinds: one kind is food pheromones, another is nest pheromones. An ant can only perceive a pheromone in the scope of its activity, and release the pheromone according to its own status.At the same time, the environment makes pheromone disappear at a certain rate.

Ants foraging environment is defined by an area under a plane rectangular coordinate system. A two-dimensional foraging space named $\mathrm{V}_{2}, \mathrm{~V}_{2}=\{\mathrm{p}(\mathrm{x}, \mathrm{y}), \mathrm{x} \in(0$, width), $\mathrm{y} \in(0$, height $), \mathrm{x}, \mathrm{y} \in \mathrm{N}\}$ : width multiplied by the height for the coordinates of the domain. The world of environment is a world of discrete, the grid is composed of line and column which stands for width and height, each grid can be described by point $\mathrm{p}(\mathrm{x}, \mathrm{y})$, as is shown in figure 1 .

Ants foraging environment still need to make environmental informations clear, like space nest, food and obstacles. The environment parameters $\operatorname{grid}(\mathrm{x}, \mathrm{y})$ is defined for the description of environmental information at each point in space, the specific meaning of parameter selection is showed in table 1.

Table 1 The parameter selection of the grid

\begin{tabular}{ccc}
\hline Variable & Values & State \\
\hline $\operatorname{grid}(\mathrm{x}, \mathrm{y})$ & 1 & obstacles \\
$\operatorname{grid}(\mathrm{x}, \mathrm{y})$ & 2 & nest \\
$\operatorname{grid}(\mathrm{x}, \mathrm{y})$ & 3 & food \\
$\operatorname{grid}(\mathrm{x}, \mathrm{y})$ & 0 & none \\
\hline
\end{tabular}

Ants make decisions according to the types and concentrations of pheromones in the environment, thus a pheromone parameter:pheromone $(\mathrm{x}, \mathrm{y})=$ ( kind,capability) is need to be defined in the environment. Pheromone $(\mathrm{x}, \mathrm{y})$ is pheromone object of $\mathrm{p}(\mathrm{x}, \mathrm{y})$. Kind represents the types of pheromones, kind $=0$ means pheromone released from ants just came out of the nest, kind $=1$ for pheromone of food. Capability represents the content of pheromone for $\mathrm{p}(\mathrm{x}, \mathrm{y})$, capability increased by the release of multiple ants, in order to limit the amount of pheromone increasing, which could lead ants to local optimum, capability would dissipate over time. The delimiter represents pheromone numerical dissipation, decided by the methods of pheromone volatilization.

\subsection{Specific steps of design model simulation}

Ant colony foraging behavior model based on agent is a method witch used the bottom-up, based on the main body interaction angle research process, the real ants foraging in the micro level on the body of the constant interaction behavior and decision model.The process of intelligent behavior emerges in the macro level. An ant only performs the following steps in each simulation step: a) initialize each variable; b) determine the scope of the perception based on effective sensing range, the perception scope determines whether find the target point or not, if it was found, then turn to i), or turn to $\mathrm{c}$ ); $\mathrm{c}$ ) randomly selected from a random number $0 \leq \mathrm{q} \leq 1$, if $\mathrm{q} \geq \mathrm{q}_{0}$, then the ant search into $\mathrm{d}$ ), otherwise the pheromone search into e); d) searching for different properties in the scope of the perception of ants, anyting there means it has found the largest amount of pheromone of ant point, turn into g), or turn to f); e)determine whether there is a perception scope pheromones or not, if there is, find the largest pheromones, turn into g), or turn to f); f)determine the main direction in the scope of perception; g)determine to move the point according to carry maximum pheromone of ant or pheromones and the main direction; h)determine the obstacles between current point and next point, if anything there, get away from this point and turn to f) to determine the new principal direction, or turn to i); i)move randomly, write down the records,spreading pheromones and volatile pheromone; j)ants that find the target would be enforced by rewards and punishment mechanism, then change its path pheromone.

In this paper, using the Java language programming model simulation. Divided it into two parts, interface and algorithm to perform simulation program, the following descriptions are the running process of the program: a) interface class instance initialization, generated a direct link between the 
instance of the class, and a map instance of a class (as the initial map); b)the environment class instance initialization, set up the ant pheromone array, instantiate the array and the static property of the control class and instances of the class respectively, set various parameters, instantiate statistical rate; c)establish the background monitoring thread, to prevent ants into a deadlock state; d) wait for the start signal; e)obtain the signal after create a thread and start; f)enter the ant behavior cycle until the stop signal detected; g)reset the ant array and statistics after a thread has stopped; h) repeat step d); i)the program ended when the user closes the interface in any steps.

\section{Ant colony foraging behavior model simulation}

\subsection{The simulation of no obstructions in the environment}

In the simulation of $300 \times 300$ two-dimensional plane, put food and nest (respective coordinates are $[20,20]$ and $[250,230]$, is located in the simulation flat of black spots in the upper left and lower right), the ant after starting from the nest, red dot marks ants, blue dot marks released pheromone of food by ants. Simulation environment without obstacles, simulation process screenshots as shown in figure $2 \sim 4$.

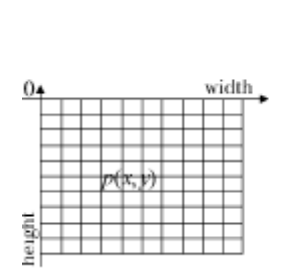

Fig. 1

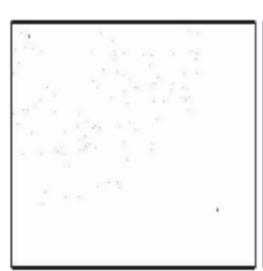

Fig. 2

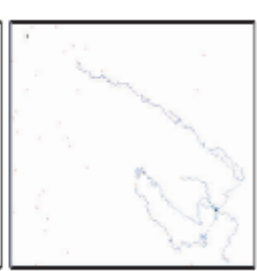

Fig. 3

In figure 2, the ant just starting from the nest, ramble in the environment randomly. After a period of time, a few ants find food source, the ants arrived at the food source, show house-hunting state immediately and spread food pheromones in the environment. Figure 3 is the first success to find the food source of ants traces of the food source of pheromone, visible traces can be used to help the ants to find the shortest path. After a number of positive feedback of ants, in the $120 \mathrm{~s}$, when the mostly ants found nests and food source between the short circuit, pheromone concentration on the route is the biggest, the ants'shortest route formed wicth can be seen from the figure 4 .

\subsection{The simulation with obstacles in environment}

Randomly set two obstacles with blue logo in the plane, it can be found that the number of free ants increases at the same simulation time, the approximation between two points is the most short circuit (figure 5), it shows that the ant in overcoming obstacles need to consume a certain amount of time. The ants' intelligence can be seen by modifing the shape of the obstacles(figure 6).

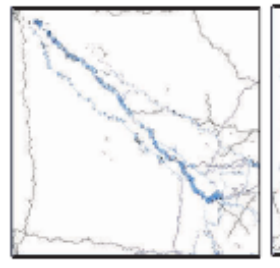

Fig. 4

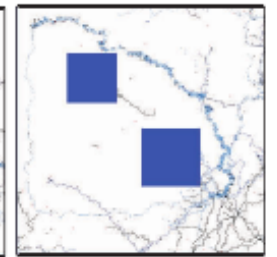

Fig. 5

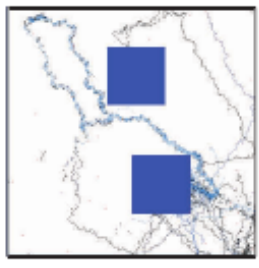

Fig. 6

\section{Model to solve local robot path planning based on foraging behavior}

\subsection{Environmental modeling}

To optimize robot path planning by using ant colony foraging model, first of all, robot working environment need to corresponding with ants foraging environment. Robots working environment should be abstracted into figure 7 world of the grid, set the starting point for the nest S, set target point $\mathrm{T}$ for food, put obstacles in environment. In this paper, the optimization of path planning guidelines for the shortest path, to find a $\mathrm{T}$ avoid the obstacles of the shortest path from $\mathrm{S}$. A robot at any grid environment can get to adjacent grids along the top, right, bottom, left, upper right, lower right, lower 
left, upper left the eight directions. Comparing figure 1 and 7, the similarity of them can be seen, so robot path planning problem can be solved by ant foraging model.

\subsection{The simulation research}

The simulation using Java programming, the size of the map is $300 \times 300$ pixels, the length of the unit is pixel, simulate the working environment with irregular obstacles.

1) Solve the optimal path of evolution under complex environment

Figure $8 \sim 10$ are ants foraging models in $300 \times 300$ size, the starting point for [20,20], [280,280], the ants look for path planning process of the shortest circuit in six straight rectangular obstruction cases. The figure $8 \sim 10$ shows that the global optimal path can be found by ant colony foraging model.

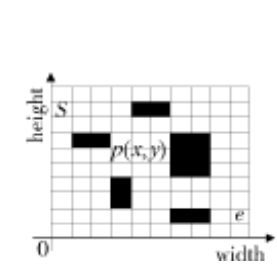

Fig. 7

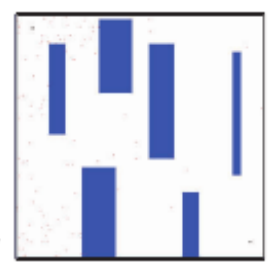

Fig. 8

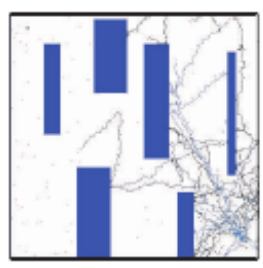

Fig. 9

2) Change the starting point and the target of the optimal path

In order to verify the ants foraging ability of solving the model, change the position of the starting point and ending point, set up the new starting point and ending point as the first two columns from table 2 , running for $180 \mathrm{~s}$, the most shortest circuit and the shortest path length and the number of ants to find the shortest path that all can be found from figure $11 \sim 14$ and table 2 .

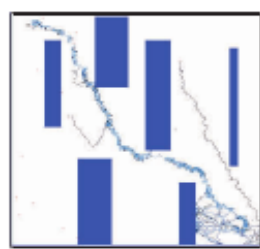

Fig. 10

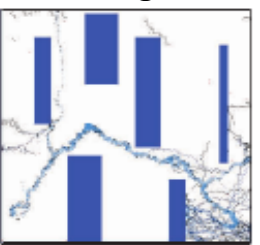

Fig. 11

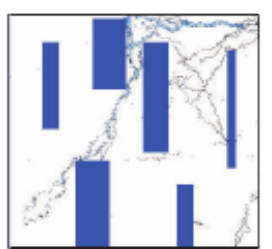

Fig. 12

Table 2 The optimal path for mobile robot

\begin{tabular}{cccccc}
\hline Start & Target & AS optimalsolution & My optimalsolution & numbers & time/s \\
\hline$[20,20]$ & {$[280,280]$} & 602 & 496 & 81 & 180 \\
{$[20,270]$} & {$[280,280]$} & 820 & 501 & 78 & 180 \\
{$[20,270]$} & {$[280,20]$} & 861 & 553 & 54 & 180 \\
{$[20,20]$} & {$[280,20]$} & 461 & 320 & 92 & 180 \\
{$[20,150]$} & {$[280,150]$} & 598 & 410 & 89 & 180 \\
\hline
\end{tabular}

As the starting point and the target point in the environment change, the ants will soon adapt to the new looking for direction and find new shortest path based on it. All of these Can be seen from the figure $11 \sim 14$ and table 2 .

3) Change the obstacle environment

Change the position of the first obstruction in the environment. After changing obstacles, new path has shown in figure 15 . When conditions change, the ants will quickly adapt to the new environment and take the short circuit based on it, as is shown from figure 15.

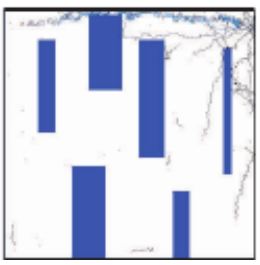

Fig. 13

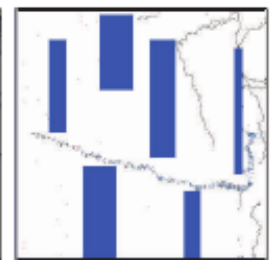

Fig. 14

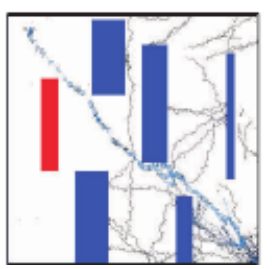

Fig. 15 
4) Compared with basic ant colony algorithm

The basic ant colony algorithm (AS) is used in the environment of robot path planning, the iterative simulation for 500 times, set the AS number $\mathrm{m}=100$, algorithm of ant information heuristic factor $\alpha=1$, expect heuristic factor $\beta=7$, pheromone volatilization coefficients $\rho=0.3$, pheromones increase intensity $\mathrm{Q}=1$. AS algorithm to search the shortest path is presented in the third column from table 2. Ant colony foraging effectiveness of the model can be seen in the third column and the fourth column in the table 2 . The simulation results show that the improved algorithm could find the optimal path with fast convergence speed, its global optimization ability and search speed are improved obviously.

\section{Conclusion}

Ant colony foraging behavior model is established in this paper by using modeling method based on principal agent of multiple rules, and finish the simulation experiments based on Java program. By comparing with a number of representative experiments, it proved that the model has strong robustness and flexibility, adaptability and fast convergence. By setting up simple rules, the use of agent of bottom-up modeling method, ant colony foraging model can be applied to the mobile robot path planning. This paper verifies that the optimal path of security can be quickly programmed and given by the ant colony foraging for food in a complicated unknown environment model, and the practicability of the ant colony foraging model can be broaden efficiently and significantly.

\section{References}

[1] Bonabeau E. Marginally stable swarms are flexible and efficient[J]. Journal de Physique I, 1996, 6(2): 309-324.

[2] JiYun Bai,Shiyong Li. Ant colony foraging behavior modeling based on agent and its application in machine into the path planning problem [J]. Computer application research, 2014, 31 (1): 47-50.

[3] Ronghua Du, Gang Yao, Quanyuan Wu. Ant colony algorithm in the application of mobile Agent migration study [J]. Journal of computer research and development, 2015, 44 (2) : 282-287.

[4] Bingde Li, Li Zhang. Improved artificial potential field method in the application of robot path planning [J]. Journal of microprocessor, 2014, 35 (5) : 51-55.

[5] Qiubo Zhong, Chao Gao, Baofu fang. Under a complex environment of humanoid robot path planning algorithm $[\mathrm{J}]$. Journal of huazhong university of science and technology: natural science edition, 2011, 39 (2) : 192-203.

[6] Juanping Zhao, Xianwen Gao, Xiuhui Fu, etc.The improved ant colony optimization algorithm of mobile robot path planning[J]. Control theory and applications, 2011, 28 (4) : 457-461.

[7] guojian Cheng, Yujia Yan, Xinjian Qian, etc. The ecological complex adaptive system modeling and simulation Based on the agents [J]. Journal of xi 'an petroleum university: natural science edition, 2011, 26 (2) : 99-103. 